DFPD-00/TH/28

\title{
Flavour non-conservation in goldstino interactions
}

\author{
Andrea Brignole由 and Anna Rossif \\ Istituto Nazionale di Fisica Nucleare, Sezione di Padova, \\ Dipartimento di Fisica 'G. Galilei', Università di Padova, \\ Via Marzolo n.8, I-35131 Padua, Italy
}

\begin{abstract}
We point out that the interactions of goldstinos with matter supermultiplets are a potential source of flavour violation, if fermion and sfermion mass matrices are not aligned and supersymmetry is spontaneously broken at a low scale. We study the impact of those couplings on low-energy processes such as $\mu \rightarrow e \gamma, \mu \rightarrow$ eee, $K \rightarrow \mu^{+} \mu^{-}, K-\bar{K}$ transitions and analogous ones. Moreover, we address the issue of flavour violation in low-energy processes involving two goldstinos and two matter fermions, generalizing earlier results obtained in the flavour-conserving case.
\end{abstract}

\footnotetext{
${ }^{1}$ E-mail address: brignole@padova.infn.it

${ }^{2}$ E-mail address: arossi@padova.infn.it
} 


\section{Introduction}

Contributions to flavour changing neutral currents (FCNC) are adequately suppressed in the standard model (SM) [1]. Supersymmetric extensions of the SM generate additional contributions to FCNC, even in the case of minimal field content and conserved R-parity (MSSM) [2]. Such effects are due to both charged and neutral couplings. In particular,

the interactions of matter multiplets with neutral gauginos, which have the form $g \tilde{f}^{*} f \lambda$, are a potential source of flavour violation that has no counterpart in the SM. Indeed, if fermion and sfermion mass matrices are not diagonal in the same superfield basis, those couplings induce flavour changing effects, which have been extensively studied (see e.g. [3, 4] and references therein). On the other hand, if fermion and sfermion mass matrices are misaligned, FCNC receive additional contributions from another class of neutral couplings: the interactions of matter multiplets with goldstinos. This observation is the starting point of the investigation we would like to present in this paper.

We recall that the supersymmetry breaking masses of the MSSM are expected to originate from the spontaneous breaking of supersymmetry in some underlying theory. This phenomenon entails the existence of a goldstone fermion, the goldstino, which couples to matter and gauge supermultiplets in a characteristic way. For instance, the interaction of a goldstino $\tilde{G}$ with a fermion-sfermion pair has the form $\left(\Delta m^{2} / F\right) \tilde{f}^{*} f \tilde{G}$, where $\sqrt{F}$ is the supersymmetry breaking scale and $\Delta m^{2}$ denotes the mass splitting in the sector under consideration [5]. The form of the interaction resembles that of a neutral gaugino, although the strength is $\Delta m^{2} / F$ instead of $g$. If the mass splitting has electroweak size whilst $\sqrt{F}$ is much larger, the goldstino is essentially decoupled. Here we are interested in the opposite scenario, in which $\sqrt{F}$ is not far from the Fermi scale $1 / \sqrt{G_{F}}$. In this case the interactions involving goldstinos are no longer negligible and can have observable effects. How to obtain low values of $\sqrt{F}$ in concrete models is an open issue, which we will not discuss. We only recall that such a possibility is not ruled out by present experiments, as shown for instance by recent studies on goldstino pair-production at $e^{+} e^{-}$and hadron colliders [7]. Therefore we believe that it is worth exploring this scenario also in connection to flavour changing phenomena, taking into account the possible flavour structure of sfermion mass matrices (that is, of $\Delta m^{2}$ ). This is the purpose of the present paper. Notice that we do not try to 'solve' the supersymmetric flavour problem, in contrast to more fundamental approaches which directly address the origin of flavour and/or supersymmetry breaking, or at least the mediation of that breaking. For instance, in models in which such mediation is due to ordinary gauge interactions (for a review, see e.g. [8]) $\sqrt{F}$ is relatively low, but still two or three orders of magnitude larger than $1 / \sqrt{G_{F}}$, so the goldstino is very weakly coupled to matter; furthermore, the mediation mechanism generates flavour-blind sfermion masses, so flavour violations through gaugino couplings are automatically suppressed in those models. Our approach here is somewhat orthogonal, i.e. phenomenological rather than model-based: we would like to study the flavour dependence of goldstino-matter interactions and the related implications for flavour changing processes in a general way, by

\footnotetext{
${ }^{1}$ In the context under consideration, the words goldstino and light gravitino can be interchanged, thanks to the equivalence theorem [5, 6]. We recall that the gravitino becomes massive by absorbing the goldstino. Its mass is related to the supersymmetry breaking and Planck scales as $m_{3 / 2}=F /\left(\sqrt{3} M_{P}\right)$. For $F \simeq \mathcal{O}\left(G_{F}^{-1}\right), m_{3 / 2} \simeq \mathcal{O}\left(10^{-5}\right) \mathrm{eV}$.
} 
treating $\sqrt{F}$ and sfermion mass matrices as free parameters, without referring to specific supersymmetry breaking or flavour models. On the phenomenological side, we will be interested in low-energy processes that only involve ordinary fermions and photons (and possibly goldstinos) as external particles?.

The paper is organized as follows. In Section 2, we embed the MSSM in an effective lagrangian in which supersymmetry is linearly realized and spontaneously broken by the auxiliary component vev of some chiral superfield $Z$, as in [9]. The couplings of the goldstino superfield $Z$ to matter and gauge superfields generate both mass and interaction terms for the component fields. In Section 3, we use those interactions to compute the rate of flavour changing radiative decays, such as $\mu \rightarrow e \gamma$ and analogous ones. In particular, we compare the contributions with goldstino exchange with the conventional ones which do not involve the goldstino multiplet. In Section 4, we discuss the generation of flavour changing effective operators with four external matter fermions, and discuss the phenomenological implications for processes such as $\mu \rightarrow e e e, K_{L} \rightarrow \mu^{+} \mu^{-}$and $K-\bar{K}$ transitions. In Section 5, we address the issue of flavour violation in processes involving two matter fermions and two goldstinos as external states. In this case, we also consider a more general approach based on the non-linear realization of supersymmetry, generalizing earlier results [10, 11]. We compare this approach with the linear one, also extending the latter to the case of mixed $F-D$ breaking. Section 6 is devoted to summary and conclusions.

\section{Supersymmetry breaking masses and goldstino couplings}

The MSSM contains quark, lepton and Higgs supermultiplets interacting with the $S U(3)_{c} \times S U(2)_{L} \times U(1)_{Y}$ vector supermultiplets. For our purposes, it will be sufficient to consider quark and charged lepton supermultiplets, coupled to the electromagnetic $U(1)_{Q}$ vector supermultiplet. The matter supermultiplets in each charge sector will be generically denoted by $E_{i}=(\tilde{f}, f)_{i}$ (charge $\left.Q_{f}\right)$ and $E_{i}^{c}=\left(\tilde{f}^{c}, f^{c}\right)_{i}\left(\right.$ charge $\left.Q_{f^{c}}=-Q_{f}\right)$, where $f=u, d, \ell$, and $i$ is a generation (i.e. flavour) index. The $U(1)_{Q}$ vector supermultiplet contains photon $\left(A_{\mu}\right)$ and photino $(\lambda)$ fields. The associated lagrangian for the component fields reads ${ }^{3}$

$$
\begin{aligned}
\mathcal{L}_{0}= & -\frac{1}{4} F_{\mu \nu} F^{\mu \nu}+i \bar{\lambda} \bar{\sigma}^{\mu} \partial_{\mu} \lambda+\frac{1}{2}(M \lambda \lambda+\text { h.c. })+\sum_{f=u, d, \ell}\left[i \bar{f} \bar{\sigma}^{\mu} D_{\mu} f+i \bar{f} \bar{\sigma}^{\mu} D_{\mu} f^{c}\right. \\
& +\left(D^{\mu} \tilde{f}\right)^{*}\left(D_{\mu} \tilde{f}\right)+\left(D^{\mu} \tilde{f}^{c}\right)^{*}\left(D_{\mu} \tilde{f}^{c}\right)+g_{e} \sqrt{2} Q_{f}\left(i \tilde{f}^{*} f \lambda-i \tilde{f}^{*} f^{c} \lambda+\text { h.c. }\right) \\
& \left.-\left(f^{c} m f+\text { h.c. }\right)-\left(\begin{array}{cc}
\tilde{f}^{*} \tilde{f}^{c}
\end{array}\right)\left(\begin{array}{cc}
m^{\dagger} m+\tilde{m}_{L L}^{2} & \tilde{m}_{L R}^{2} \\
\tilde{m}_{R L}^{2} & m m^{\dagger}+\tilde{m}_{R R}^{2}
\end{array}\right)\left(\begin{array}{c}
\tilde{f} \\
\tilde{f}^{*}
\end{array}\right)\right]+\ldots(1)
\end{aligned}
$$

\footnotetext{
${ }^{2}$ If sfermions are also allowed to be external, other processes could be considered. The flavour changing decays $\tilde{f}_{i} \rightarrow f_{j} \tilde{G}$ are obvious examples.

${ }^{3}$ We use two component spinor notation, with $\sigma^{\mu} \equiv(1, \vec{\sigma}), \bar{\sigma}^{\mu} \equiv(1,-\vec{\sigma}), \sigma^{\mu \nu} \equiv \frac{1}{4}\left(\sigma^{\mu} \bar{\sigma}^{\nu}-\sigma^{\nu} \bar{\sigma}^{\mu}\right)$, $\bar{\sigma}^{\mu \nu} \equiv \frac{1}{4}\left(\bar{\sigma}^{\mu} \sigma^{\nu}-\bar{\sigma}^{\nu} \sigma^{\mu}\right)$ and $g_{\mu \nu}=\operatorname{diag}(+1,-1,-1,-1)$. We recall that $f$ and $\bar{f}^{c}$ correspond to the left and right components of the four component Dirac spinor $\Psi_{f}$, whereas $\tilde{f}$ and $\tilde{f}^{c}{ }^{*}$ correspond to the fields usually denoted as $\tilde{f}_{L}$ and $\tilde{f}_{R}$.
} 
where $g_{e}$ is the electromagnetic gauge coupling, $D_{\mu}=\partial_{\mu}+i g_{e} A_{\mu} Q$ and the dots denote sfermion self-interactions. Note that $m, \tilde{m}_{L L}^{2}, \tilde{m}_{R R}^{2}, \tilde{m}_{R L}^{2}, \tilde{m}_{L R}^{2}=\left(\tilde{m}_{R L}^{2}\right)^{\dagger}$ are $3 \times 3$ matrices in each charged sector and should be labelled by an index $f$. Both this index and generation indices are understood for simplicityf. If fermion and sfermion mass matrices are not diagonal in the same superfield basis, flavour changing effects arise through the gaugino-fermion-sfermion vertices. Notice that we do not assume that the matrix $\tilde{m}_{R L}^{2}$ is proportional to the matrix $m$. However, in order to simplify the power counting in the next sections, we will make the reasonable assumption that both matrices have a common chiral suppression. Thus, in the fermion mass basis, the diagonal entries of $\tilde{m}_{R L}^{2}$ are expected to be of order 'supersymmetry breaking mass' $\times$ 'appropriate fermion mass', and a further (model dependent) suppression factor can be expected in the off-diagonal entries.

Now we have to specify how the matter and gauge multiplets couple to the goldstino, without relying on some specific fundamental mechanism for supersymmetry breaking. This can be done in several ways. For instance, the interactions between one goldstino and a fermion-sfermion pair, which are model-independent, can easily be derived from supercurrent conservation [5]. For interactions involving more than one goldstino, other methods have to be used. Here we follow the approach of ref. [9], where the spontaneous breaking of supersymmetry is described at an effective level. Therefore we will consider the above lagrangian (1) as part of an effective globally supersymmetric lagrangian in which the matter and gauge superfields are also coupled to some neutral chiral superfield $Z$. Supersymmetry, which is linearly realized, is assumed to be spontaneously broken by the auxiliary component of $Z$, through a non-vanishing expectation value $\left\langle\left. Z\right|_{\theta \theta}>=-F\right.$. The mass parameter $\sqrt{F}$ is the supersymmetry breaking scale. The physical components in $Z$ are a Weyl fermion, namely the goldstino $\tilde{G}$, and a complex scalar $z$, called sgoldstinof. The effective couplings between the goldstino superfield $Z$ and matter or gauge superfields generate not only the supersymmetry breaking masses shown in $\mathcal{L}_{0}$, through the above vev, but also closely related interactions, to be illustrated now.

Matter fermion masses, as well as the associated 'supersymmetric' sfermion masses, can be derived from superpotential terms of the form $E^{c} m E$. Consider now the supersymmetry breaking mass matrices in the sfermion sector, i.e. $\tilde{m}_{L L}^{2}, \tilde{m}_{R R}^{2}$ and $\tilde{m}_{R L}^{2}$. The $L L(R R)$ mass terms can be derived from Kähler potential terms of the form $|Z|^{2} E^{*} E\left(|Z|^{2} E^{c} E^{c *}\right)$, suppressed by the square of some scale $\tilde{\Lambda}$, whereas the $R L$ mass terms can be derived from superpotential terms of the form $Z E^{c} E$. All such terms contain arbitrary dimensionless flavour matrices. Since these matrices are in one-to-one correspondence with the matrices $\tilde{m}_{L L}^{2}, \tilde{m}_{R R}^{2}$ and $\tilde{m}_{R L}^{2}$, we will trade the former set of parameters (plus $\tilde{\Lambda}$ ) for the latter set of physical parameters (including $\sqrt{F}$ ). Those Kähler potential and superpotential terms generate not only masses, but also several interactions (of dimension 4 or higher) involving the physical components of the matter and goldstino superfields 0 . In particular,

4 The inclusion of neutrinos requires minor modifications. Consider the case in which only $\nu$ and $\tilde{\nu}$ are present in the low-energy theory. Then, wherever a sum $\sum_{f}$ appears or is understood, the neutrino contributions can be formally obtained by putting $f=f^{c}=\nu, \tilde{f}=\tilde{f} c=\tilde{\nu}, \tilde{m}_{L L}^{2}=\tilde{m}_{R R}^{2}$ and multiplying by a factor $1 / 2$. For instance, the neutrino and sneutrino mass terms in eq. (1) would read as $-\frac{1}{2}(\nu m \nu+$ h.c.) and $-\tilde{\nu}^{*}\left(m^{\dagger} m+\tilde{m}_{L L}^{2}\right) \tilde{\nu}-\frac{1}{2}\left(\tilde{\nu} \tilde{m}_{R L}^{2} \tilde{\nu}+\right.$ h.c. $)$.

${ }^{5}$ We work in field coordinates such that $\langle z\rangle=0,\left\langle K_{\bar{z} z}\right\rangle=1$ and the parameters $F$ and $M$ are real and positive.

6 The effective supersymmetric lagrangian could contain other superfield interactions besides those 
the following cubic interactions emerge:

$$
\begin{gathered}
-\frac{1}{F} \sum_{f}\left(\begin{array}{cc}
\tilde{f}^{*} & \tilde{f}^{c}
\end{array}\right)\left(\begin{array}{cc}
\tilde{m}_{L L}^{2} & \tilde{m}_{L R}^{2} \\
\tilde{m}_{R L}^{2} & \tilde{m}_{R R}^{2}
\end{array}\right)\left(\begin{array}{c}
f \tilde{G} \\
\bar{f}^{c} \tilde{G}
\end{array}\right)+\text { h.c. } \\
-\frac{1}{F} \sum_{f} z f^{c} \tilde{m}_{R L}^{2} f+\text { h.c. }
\end{gathered}
$$

Among quartic interactions, the following ones will be relevant for our purposes:

$$
-\frac{1}{2 F^{2}}\left[\overline{\tilde{G}} \bar{\sigma}_{\mu} \tilde{G}+i\left(z^{*} \partial_{\mu} z-z \partial_{\mu} z^{*}\right)\right] \sum_{f}\left(\bar{f} \tilde{m}_{L L}^{2} \bar{\sigma}^{\mu} f-f^{c} \tilde{m}_{R R}^{2} \sigma^{\mu} \bar{f}^{c}\right)
$$

We stress again that the interactions of a goldstino with fermion-sfermion bilinears in (2) are completely model independent, as one can easily check by using supercurrent conservation. As already mentioned, such interactions resemble those of a neutral gaugino, with $\tilde{m}^{2} / F$ playing the role of $g$ [5]. Notice however that, in contrast to the case of gauginos, here the coupling already has a flavour structure. However, this is not a new independent structure: it is uniquely specified by the mass matrices, which also dictate the subsequent rotation to the physical bases. So the interactions of neutral gauginos and those of goldstinos are expected to have a similar impact on FCNC processes, once the parameter mapping is taken into account. Nevertheless, the fact that the goldstino is the goldstone particle of spontaneously broken supersymmetry makes it quite special. The peculiar low-energy properties of goldstinos will especially emerge in our final section.

As regards the gaugino mass term in $\mathcal{L}_{0}$, one can effectively derive it from a superfield coupling of the form $(\eta / \tilde{\Lambda}) Z \mathcal{W W}$ (where $\mathcal{W}$ is the gauge superfield strength), i.e. from a linear term in the gauge kinetic function. The associated interactions involving the physical components of the vector and goldstino superfields can be written in terms of $M$ and $F[9]$ :

$$
-\frac{M}{\sqrt{2} F} \tilde{G} \sigma^{\mu \nu} \lambda F_{\mu \nu}-\frac{M}{4 F} z\left(F_{\mu \nu} F^{\mu \nu}+i F_{\mu \nu} \tilde{F}^{\mu \nu}\right)+\text { h.c. }+\ldots
$$

where $\tilde{F}^{\mu \nu}=\frac{1}{2} \epsilon^{\mu \nu \rho \sigma} F_{\rho \sigma}$ and the dots stand for terms which we will not need. Other superpotential and Kähler potential terms generate kinetic, mass and interaction terms in the $Z$ sector [9]:

$$
\begin{aligned}
& i \overline{\widetilde{G}} \bar{\sigma}^{\mu} \partial_{\mu} \tilde{G}+\frac{1}{2}\left(\partial^{\mu} S \partial_{\mu} S-m_{S}^{2} S^{2}\right)+\frac{1}{2}\left(\partial^{\mu} P \partial_{\mu} P-m_{P}^{2} P^{2}\right) \\
& -\frac{1}{2 \sqrt{2} F}\left(m_{S}^{2} S \tilde{G} \tilde{G}-i m_{P}^{2} P \tilde{G} \tilde{G}+\text { h.c. }\right)-\frac{m_{S}^{2}+m_{P}^{2}}{8 F^{2}} \tilde{G} \tilde{G} \bar{G} \bar{G}+\ldots
\end{aligned}
$$

We have assumed for simplicity that the mass eigenstates in the sgoldstino sector coincide with the real and imaginary parts of $z=(S+i P) / \sqrt{2}$. We will also assume that sgoldstino masses are not much lighter than squark and slepton masses, as suggested by naturalness

considered here. Some of them can be eliminated in favour of the existing ones through field redefinitions. Other ones depend on additional arbitrary parameters, not directly related to the mass spectrum. We will not discuss them, since we choose to focus on couplings that are related to the mass spectrum. 
considerations [12]. If this assumption is relaxed and sgoldstinos are allowed to be very light, enhancement effects can appear in several processes (see e.g. [13, 14]), including some FCNC processes to be discussed below. However, in such cases $\sqrt{F}$ is typically forced to be substantially larger than the electroweak scale, which is not the scenario we would like to study】.

\section{Flavour changing radiative decays}

In this section we will discuss flavour changing radiative decays. For definiteness, we focus on the decay $\mu^{-} \rightarrow e^{-} \gamma$, which violates individual lepton flavours. The effective operator responsible for such a decay can be parametrized as

$$
\mathcal{L}_{e f f}=\frac{i g_{e}}{16 \pi^{2}} m_{\mu}\left(C_{R} \bar{e} \bar{\sigma}^{\mu \nu} \bar{\mu}^{c}+C_{L} e^{c} \sigma^{\mu \nu} \mu\right) F_{\mu \nu}
$$

This leads to the branching ratio

$$
B R(\mu \rightarrow e \gamma)=\frac{3 \alpha}{16 \pi G_{F}^{2}}\left(\left|C_{R}\right|^{2}+\left|C_{L}\right|^{2}\right) B R\left(\mu \rightarrow e \nu_{\mu} \bar{\nu}_{e}\right)
$$

where $B R\left(\mu \rightarrow e \nu_{\mu} \bar{\nu}_{e}\right) \simeq 1$.

The interactions involving goldstinos and sgoldstinos described in the previous section generate several one-loop contributions to $C_{R}$ and $C_{L}$, through the diagrams schematically shown in Fig. 1. We disregard type (d) diagrams, since they are quadratic in $\tilde{m}_{L R}^{2}$ and we

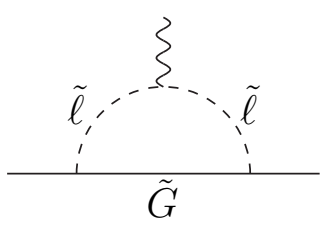

(a)

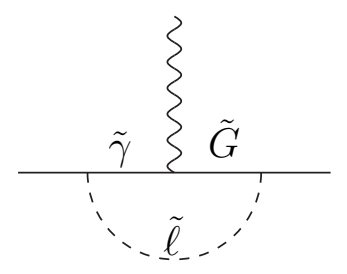

(b)

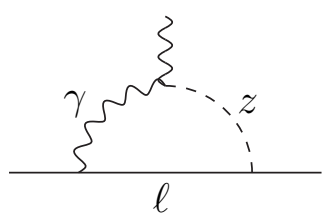

$(c)$

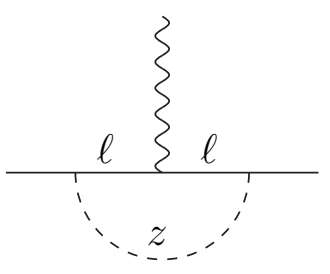

$(d)$

Figure 1: Diagrams with goldstino or sgoldstino exchange contributing to $\mu \rightarrow e \gamma$.

are only working at first order in the muon mass. From the other diagrams, we obtain:

$$
\begin{aligned}
& C_{R}^{(G)}=\frac{1}{2 F^{2}}\left[-\frac{1}{6} \tilde{m}_{L L}^{2}+\left(\frac{\tilde{\mathbf{m}}^{2} M^{2}}{\tilde{\mathbf{m}}^{2}-M^{2}}\left(1-\frac{M^{2}}{\tilde{\mathbf{m}}^{2}-M^{2}} \log \frac{\tilde{\mathbf{m}}^{2}}{M^{2}}\right)\right)_{L L}+\left(\log \frac{m_{P}^{2}}{m_{S}^{2}}\right) \frac{M}{m_{\mu}} \tilde{m}_{L R}^{2}\right]_{e \mu}(9) \\
& C_{L}^{(G)}=\frac{1}{2 F^{2}}\left[-\frac{1}{6} \tilde{m}_{R R}^{2}+\left(\frac{\tilde{\mathbf{m}}^{2} M^{2}}{\tilde{\mathbf{m}}^{2}-M^{2}}\left(1-\frac{M^{2}}{\tilde{\mathbf{m}}^{2}-M^{2}} \log \frac{\tilde{\mathbf{m}}^{2}}{M^{2}}\right)\right)_{R R}+\left(\log \frac{m_{P}^{2}}{m_{S}^{2}}\right) \frac{M}{m_{\mu}} \tilde{m}_{R L}^{2}\right]_{e \mu}(10)
\end{aligned}
$$

where $\tilde{\mathbf{m}}^{2}$ stands for the $6 \times 6$ slepton mass matrix, whose $3 \times 3$ blocks are $\tilde{m}_{L L}^{2}, \tilde{m}_{R R}^{2}, \tilde{m}_{L R}^{2}$, $\tilde{m}_{R L}^{2}$. These results generalize to flavour-changing transitions those obtained for diagonal

\footnotetext{
${ }^{7}$ Collider signals of massive sgoldstinos in the case of low $\sqrt{F}$ have been recently analysed in 15$]$.
} 
magnetic moments in the absence of flavour mixing [16, 14. The above expressions hold in the superfield basis in which leptons are mass eigenstates. The computation can be performed by using matrix vertices and propagators in that basis. Alternatively, one can diagonalize the slepton mass matrix as well and move back to the other basis in the end. The three terms in each expression originate from diagrams of type (a), type (b) and type (c), respectively. Notice that type (a) contributions are simply proportional to the $e \mu$ element of the matrices $\tilde{m}_{L L}^{2}$ and $\tilde{m}_{R R}^{2}$. The latter result holds for arbitrary $\tilde{\mathbf{m}}^{2}$ : it does not rely on any assumption on the size of the off-diagonal entries of $\tilde{m}_{L L}^{2}$ and $\tilde{m}_{R R}^{2}$, or even on the assumption that the entries of $\tilde{m}_{R L}^{2}$ are linear in lepton masses. The matrix structure of vertices and propagators just combine in the proper way, and the final result turns out to coincide with what we would have obtained in the simple mass insertion approximation $^{8}$. Type (c) contributions are proportional to the $e \mu$ elements of $\tilde{m}_{L R}^{2}$ and $\tilde{m}_{R L}^{2}$, but here the reason is more obvious. On the other hand, type (b) contributions are expressed through a non-trivial function of the matrix $\tilde{\mathbf{m}}^{2}$. In this case, in order to obtain an approximate expression, we can expand $\tilde{\mathbf{m}}^{2}$ around the diagonall and work to first order in the flavour changing elements of $\tilde{\mathbf{m}}^{2}$, now assumed to be small. This corresponds to the mass-insertion approximation and allows us to cast type (b) contributions in a form similar to the other ones. Under this approximation, we can rewrite $C_{R}^{(G)}$ and $C_{L}^{(G)}$ as

$$
\begin{aligned}
C_{R}^{(G)} & =\frac{1}{F^{2}}\left[H_{1}\left(\frac{M^{2}}{\tilde{m}^{2}}\right)\left(\tilde{m}_{L L}^{2}\right)_{e \mu}+\frac{1}{2}\left(\log \frac{m_{P}^{2}}{m_{S}^{2}}\right) \frac{M\left(\tilde{m}_{L R}^{2}\right)_{e \mu}}{m_{\mu}}\right] \\
C_{L}^{(G)} & =\frac{1}{F^{2}}\left[H_{1}\left(\frac{M^{2}}{\tilde{m}^{2}}\right)\left(\tilde{m}_{R R}^{2}\right)_{e \mu}+\frac{1}{2}\left(\log \frac{m_{P}^{2}}{m_{S}^{2}}\right) \frac{M\left(\tilde{m}_{R L}^{2}\right)_{e \mu}}{m_{\mu}}\right]
\end{aligned}
$$

where

$$
H_{1}(x)=\frac{-1+3 x-15 x^{2}+13 x^{3}-6 x^{2}(1+x) \log x}{12(1-x)^{3}}
$$

The function $H_{1}(x)$ is negative for $x<1$ and positive for $x>1$. Notice that $H_{1}(1)=0$ : when $\tilde{m}^{2}=M^{2}$, type (a) and type (b) contributions cancel each other, to linear order in the flavour changing masses.

We would like to compare the above contributions, which we will simply call 'goldstino contributions', to the more conventional non-goldstino contributions. In the full MSSM, the latter ones arise from both charged and neutral interactions. The reference lagrangian $\mathcal{L}_{0}$ only gives neutral contributions, from type (a) diagrams in which the goldstino is replaced by a photino. In contrast to the goldstino, however, the photino propagator can either conserve or flip chirality, so several contributions arise. In the mass-insertion approximation, we find (in agreement with [17, 3]):

$$
C_{R}^{(0)}=\frac{2 g_{e}^{2}}{\tilde{m}^{4}}\left[\left(H_{2}\left(\frac{M^{2}}{\tilde{m}^{2}}\right)+H_{3}\left(\frac{M^{2}}{\tilde{m}^{2}}\right) \frac{M\left(\tilde{m}_{L R}^{2}\right)_{\mu \mu}}{\tilde{m}^{2} m_{\mu}}\right)\left(\tilde{m}_{L L}^{2}\right)_{e \mu}-H_{4}\left(\frac{M^{2}}{\tilde{m}^{2}}\right) \frac{M\left(\tilde{m}_{L R}^{2}\right)_{e \mu}}{m_{\mu}}\right]
$$

${ }^{8}$ Incidentally, we remark that even the mass insertion method has new features in the present context. For type (a) diagrams, for instance, the flavour violating factor $\left(\tilde{m}_{L L}^{2}\right)_{e \mu}$ (or $\left.\left(\tilde{m}_{R R}^{2}\right)_{e \mu}\right)$ can be inserted in either a slepton propagator or a lepton-slepton-goldstino vertex. Moreover, the vertex contributions are twice as large as the propagator contributions and have opposite sign.

${ }^{9}$ For simplicity, we will consider the diagonal entries of $\tilde{\mathbf{m}}^{2}$ to have a common value $\tilde{m}^{2}$. The generalization is straightforward. 


$$
C_{L}^{(0)}=\frac{2 g_{e}^{2}}{\tilde{m}^{4}}\left[\left(H_{2}\left(\frac{M^{2}}{\tilde{m}^{2}}\right)+H_{3}\left(\frac{M^{2}}{\tilde{m}^{2}}\right) \frac{M\left(\tilde{m}_{R L}^{2}\right)_{\mu \mu}}{\tilde{m}^{2} m_{\mu}}\right)\left(\tilde{m}_{R R}^{2}\right)_{e \mu}-H_{4}\left(\frac{M^{2}}{\tilde{m}^{2}}\right) \frac{M\left(\tilde{m}_{R L}^{2}\right)_{e \mu}}{m_{\mu}}\right]
$$

where the loop functions $H_{2}(x), H_{3}(x), H_{4}(x)$ are

$$
\begin{aligned}
& H_{2}(x)=\frac{1-9 x-9 x^{2}+17 x^{3}-6 x^{2}(3+x) \log x}{12(1-x)^{5}} \\
& H_{3}(x)=\frac{1+9 x-9 x^{2}-x^{3}+6 x(1+x) \log x}{2(1-x)^{5}} \\
& H_{4}(x)=\frac{1+4 x-5 x^{2}+2 x(2+x) \log x}{2(1-x)^{4}}
\end{aligned}
$$

Goldstino and non-goldstino contributions exhibit a similar structure. They are proportional to the flavour changing $(e \mu)$ elements of the slepton mass matrix, depend on dimensionless functions of superpartner masses and are suppressed by the fourth power of some scale. This scale is the supersymmetry breaking scale for the goldstino contributions and a supersymmetry breaking mass (e.g. the average slepton mass $\tilde{m}$ ) for the nongoldstino contributions. If $\sqrt{F}$ is much larger than the supersymmetry breaking masses, the goldstino contributions are negligible in comparison to the non-goldstino ones. On the other hand, if $\sqrt{F}$ and the supersymmetry breaking masses have a similar size, then goldstino and non-goldstino diagrams give similar contributions to $\mu \rightarrow e \gamma$. It is interesting to make a more quantitative comparison. For definiteness, we neglect LR terms (both flavour conserving and flavour changing ones) and focus on the contributions proportional to $\left(\tilde{m}_{L L}^{2}\right)_{e \mu}$, i.e. we consider

$$
C_{R}^{(G)}=\frac{1}{F^{2}} H_{1}\left(\frac{M^{2}}{\tilde{m}^{2}}\right)\left(\tilde{m}_{L L}^{2}\right)_{e \mu}, \quad C_{R}^{(0)}=\frac{2 g_{e}^{2}}{\tilde{m}^{4}} H_{2}\left(\frac{M^{2}}{\tilde{m}^{2}}\right)\left(\tilde{m}_{L L}^{2}\right)_{e \mu}
$$

We recall that $H_{1}(x)$ can have either sign, whereas $H_{2}(x)$ is positive. To measure the relative importance of the goldstino contributions versus the non-goldstino ones, we introduce the ratio

$$
R=\frac{C_{R}^{(G)}}{C_{R}^{(0)}}=\frac{\tilde{m}^{4}}{2 g_{e}^{2} F^{2}} \frac{H_{1}\left(\frac{M^{2}}{\tilde{m}^{2}}\right)}{H_{2}\left(\frac{M^{2}}{\tilde{m}^{2}}\right)}
$$

which does not depend on $\left(\tilde{m}_{L L}^{2}\right)_{e \mu}$. In the limit $\tilde{m} \gg M$, for instance, that ratio is $R=-\tilde{m}^{4} /\left(2 g_{e}^{2} F^{2}\right)$, which becomes -1 when $\tilde{m} \simeq 0.65 \sqrt{F}$. Contours of $R$ in the $(\tilde{m} / \sqrt{F}, M / \sqrt{F})$ plane are shown in Fig. 2. Goldstino contributions are smaller (larger) than the other ones in the region with $|R|<1(|R|>1)$. We can also combine the two classes of contributions and study $B R(\mu \rightarrow e \gamma)$ as a function of $\tilde{m}, M, \sqrt{F}$ and $\left(\delta_{L L}\right)_{e \mu} \equiv\left(\tilde{m}_{L L}^{2}\right)_{e \mu} / \tilde{m}^{2}$. In Fig. 3 we have fixed some representative values of $(\tilde{m}, M)$ and shown the lines in the $\left(\sqrt{F},\left|\left(\delta_{L L}\right)_{e \mu}\right|\right)$ plane along which $B R(\mu \rightarrow e \gamma)$ saturates the present experimental bound, which is $1.2 \times 10^{-11}$ [18]. In other words, the lines give the upper bound on $\left|\left(\delta_{L L}\right)_{e \mu}\right|$ as a function of $\sqrt{F}$. When $\sqrt{F}$ is large such bounds are determined by the (conventional) non-goldstino contributions. When $\sqrt{F}$ decreases, the latter contributions start to interfere with the goldstino ones: for $\tilde{m}>M$ the interference is destructive, the bound on $\left|\left(\delta_{L L}\right)_{e \mu}\right|$ tends to disappear and the curves exhibit a peak, 


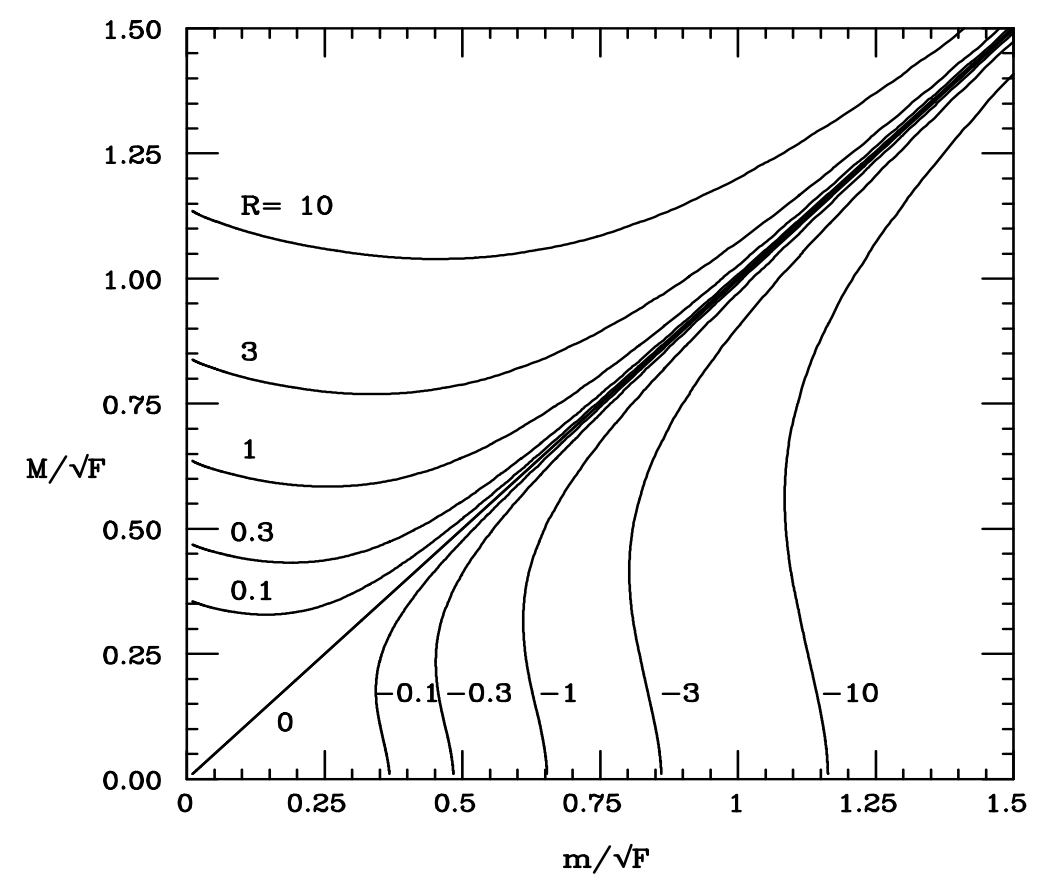

Figure 2: The ratio of goldstino versus non-goldstino contributions to the $\mu \rightarrow$ er amplitude in the $(\tilde{m} / \sqrt{F}, M / \sqrt{F})$ plane.

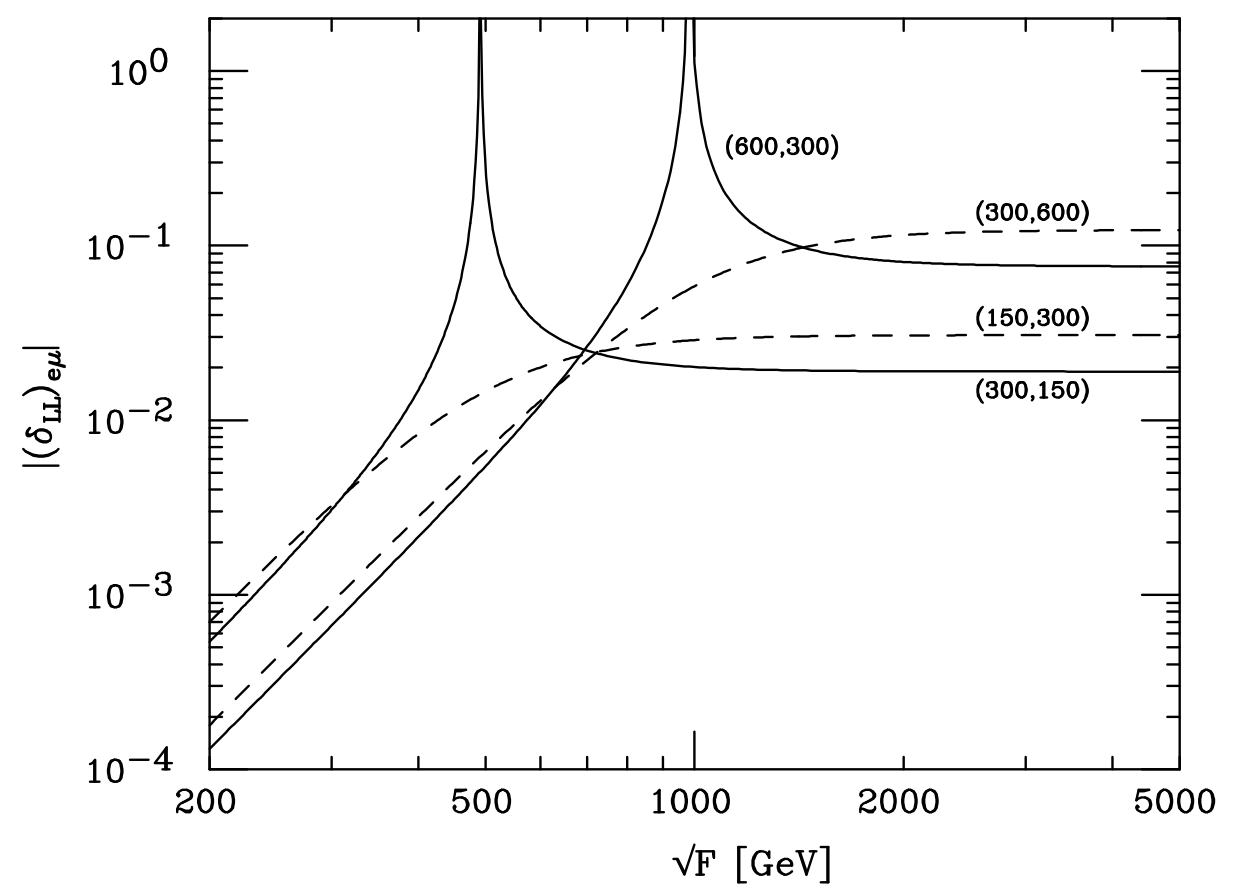

Figure 3: Lines of constant $B R(\mu \rightarrow e \gamma)=1.2 \times 10^{-11}$ in the $\left(\sqrt{F},\left|\left(\delta_{L L}\right)_{e \mu}\right|\right)$ plane, for different choices of $(\tilde{m}, M)[\mathrm{GeV}]$. 
whereas for $\tilde{m}<M$ the interference is constructive and the curves show a knee. For even smaller values of $\sqrt{F}$, i.e. to the left of the transition region, the goldstino contributions dominate and the bounds on $\left|\left(\delta_{L L}\right)_{e \mu}\right|$ become stronger than the conventional ones. In the limit $\tilde{m} \gg M$, for instance, the bound from goldstino contributions alone can be written as

$$
\left|\left(\delta_{L L}\right)_{e \mu}\right| \lesssim 2 \times 10^{-3}\left(\frac{\sqrt{F}}{\tilde{m}}\right)^{4}\left(\frac{\tilde{m}}{300 \mathrm{GeV}}\right)^{2}
$$

An identical discussion applies to the contributions that depend on $\left(\delta_{R R}\right)_{e \mu}$.

It is straightforward to translate the above discussion to the decays $\tau \rightarrow e \gamma$ and $\tau \rightarrow \mu \gamma$, whose branching ratios are experimentally bounded by $2.7 \times 10^{-6}$ [19 and $1.1 \times 10^{-6}$ [20], respectively. Notice that Fig. 2 applies to these cases as well, whereas in Fig. 3 only the scale of the vertical axis has to be changed: $\left|\left(\delta_{L L}\right)_{e \mu}\right|$ has to be replaced by either $10^{-3}\left|\left(\delta_{L L}\right)_{e \tau}\right|$ or $1.4 \times 10^{-3}\left|\left(\delta_{L L}\right)_{\mu \tau}\right|$. Therefore the qualitative description remains the same as before, although the constraints on $\left|\left(\delta_{L L}\right)_{e \tau}\right|$ and $\left|\left(\delta_{L L}\right)_{\mu \tau}\right|$ are of course much weaker.

The above discussion can also be extended to flavour changing transitions in the quark sector, such as $b \rightarrow s \gamma$. This decay is potentially sensitive to the $b s$ entries of the down squark mass matrix, through both non-goldstino and goldstino contributions. The former ones are mainly due to squark-gluino (rather than squark-photino) exchange [21, 3]. The latter ones become comparable to those when $\tilde{m}^{2} / F \sim g_{s}$, where $g_{s}$ is the strong coupling constant and $\tilde{m}$ is an average squark mass. However, neither contribution gives significant constraints.

\section{Flavour changing processes with four matter fermions}

We now discuss the FCNC processes that involve four matter fermions as external states. In both the SM and the MSSM, the leading perturbative contributions to such processes generically arise at one-loop level, and are finite. If goldstino and sgoldstino couplings are also taken into account, additional contributions arise.

Some contributions arise already at tree level. Indeed, when two $z f f^{c}$ vertices are joined by a sgoldstino propagator, effective four fermion interactions are generated:

$$
\mathcal{L}_{\text {eff }}=\frac{1}{4 F^{2}}\left[\frac{1}{m_{S}^{2}}\left(\sum_{f} f^{c} \tilde{m}_{R L}^{2} f+\text { h.c. }\right)^{2}+\frac{1}{m_{P}^{2}}\left(i \sum_{f} f^{c} \tilde{m}_{R L}^{2} f+\text { h.c. }\right)^{2}\right]
$$

However, owing to the assumed chiral suppression of $\tilde{m}_{R L}^{2}$ (and the assumed size of sgoldstino masses), the coefficients of these four fermion operators are at most $\mathcal{O}\left(m_{f}^{2} / F^{2}\right) \lesssim$ $\mathcal{O}\left(m_{f}^{2} G_{F}^{2}\right)$, i.e. they are automatically suppressed by fermion masses巴. On the other

${ }^{10}$ Incidentally, we recall that perturbativity considerations require that the ratio $\tilde{m} / \sqrt{F}$ be smaller than $2 \div 3$ 9, 12]: this should be understood everywhere. We also notice that the inequality (21) can be equivalently written as $\left(\tilde{m}_{L L}^{2}\right)_{e \mu} \lesssim(13 \mathrm{GeV})^{2}(\sqrt{F} / 300 \mathrm{GeV})^{4}$, i.e. $\tilde{m}^{2}$ drops out. This example shows that, in the case of goldstino contributions, the parametrization in terms of $\left(\delta_{L L}\right)_{e \mu}$ and $\tilde{m}^{2}$ may be redundant. We adopt it to allow for an easier comparison with the literature.

${ }^{11}$ For a similar reason, other interactions due to tree-level sgoldstino exchange are also suppressed. For 
hand, if the above mentioned assumptions are relaxed, enhancement effects can appear: in this case, the required suppression should be provided by large values of $\sqrt{F}$ and/or intrinsically small flavour violation in $\tilde{m}_{R L}^{2}$.

We would like to discuss contributions to four fermion processes that are not chirally suppressed, so we neglect $\tilde{m}_{R L}^{2}$ and move to one-loop level. In analogy to the SM or the MSSM, several one-loop diagrams contribute, both 1PI (e.g. boxes) and 1PR (e.g. penguins). Some 1PI diagrams are skecthed in Figure 4. Diagram (a) is a non-goldstino MSSM diagram, which we show for comparison: it is a typical gaugino-sfermion box. By replacing gaugino-fermion-sfermion vertices with goldstino-fermion-sfermion vertices, we obtain box diagrams like (b) and (c). All these boxes give finite contributions ${ }^{\mathrm{T2}}$. In our supersymmetric effective lagrangian, however, goldstinos (and sgoldstinos) also couple to matter through non-renormalizable couplings. For example, the theory contains quartic interactions of dimension six, like those in eq. (㺼, whose coefficients are again determined by sfermion masses and $\sqrt{F}$. These interactions cannot be simply dropped, since they play a crucial role in the low-energy cancellations that take place in diagrams with external goldstinos (see next section). Such vertices can be used to build new (non box) 1PI diagrams, like (d), (e), (f) in Figure 4. These diagrams are not finite: the dependence on the cutoff scale is logarithmic for diagram (d) and quadratic for diagrams (e) and (f). Thus, in contrast to what happens in the SM or the MSSM, flavour changing

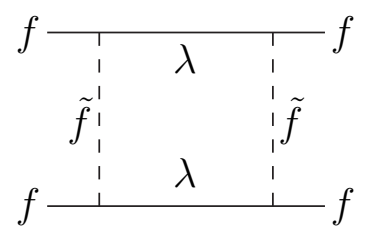

(a)

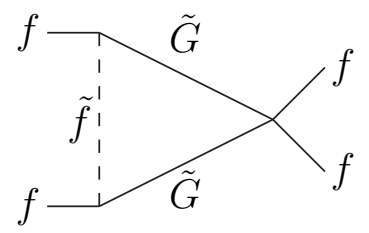

$(d)$

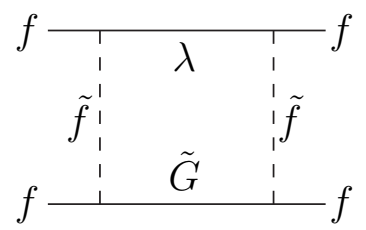

(b)

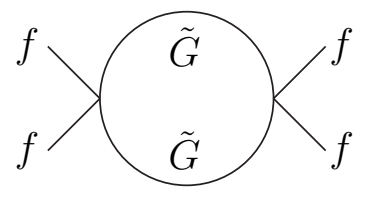

$(e)$

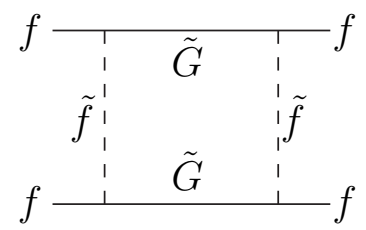

$(c)$

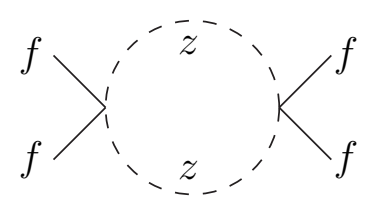

$(f)$

Figure 4: Examples of one-particle-irreducible contributions to flavour changing four fermion operators. The symbols $f$ and $\tilde{f}$ generically denote matter fermions and sfermions. Flavour changes can occur in sfermion propagators, cubic vertices or quartic vertices.

four fermion interactions receive both finite and divergent contributions in the effective instance, by connecting a $z f f^{c}$ vertex with a $z \gamma \gamma$ vertex, we obtain the effective (two-fermion)-(twophoton) coupling $\frac{M}{4 F^{2}}\left(\sum_{f} f^{c} \tilde{m}_{R L}^{2} f\right)\left(\frac{1}{m_{S}^{2}} F_{\mu \nu} F^{\mu \nu}-\frac{i}{m_{P}^{2}} F_{\mu \nu} \tilde{F}^{\mu \nu}\right)+$ h.c., which could contribute e.g. to the decay $\mu \rightarrow e \gamma \gamma$.

${ }^{12}$ For instance, the coefficients of $\Delta F=1$ four fermion operators induced by (a), (b) and (c) scale as $\left(g^{4} / \tilde{m}^{4}\right) \tilde{m}_{i j}^{2},\left(g^{2} / F^{2}\right) \tilde{m}_{i j}^{2}$ and $\left(\tilde{m}^{4} / F^{4}\right) \tilde{m}_{i j}^{2}$, respectively, where $\tilde{m}_{i j}^{2}$ is the appropriate flavour changing sfermion mass. 
theory considered here. In principle, such divergences could be reabsorbed by introducing other terms in the supersymmetric effective lagrangian, such as (for instance) Kähler potential terms quartic in the matter superfields. These new terms would not only act as counterterms, but also generate new contributions. Strictly speaking, all this means that the coefficients of four fermion interactions cannot be predicted in terms of the parameters already introduced [3]. However, we can also adopt the milder point of view that the one-loop diagrams generated by the interactions originally introduced give naturalness estimates of the coefficients of four-fermion interactions, once the cutoff scale is specified ${ }^{\text {Tt }}$. We continue the discussion in this spirit and focus on the contributions generated by diagrams (e) and (f) in Fig. 4. By retaining only the quadratic dependence on the cutoff scale $\Lambda$, we obtain:

$$
\mathcal{L}_{e f f}=-\frac{1}{64 \pi^{2}} \frac{\Lambda^{2}}{F^{4}}\left[\sum_{f}\left(\bar{f} \tilde{m}_{L L}^{2} \bar{\sigma}^{\mu} f-f^{c} \tilde{m}_{R R}^{2} \sigma^{\mu} \bar{f}^{c}\right)\right]\left[\sum_{f^{\prime}}\left(\bar{f}^{\prime} \tilde{m}_{L L}^{2} \bar{\sigma}_{\mu} f^{\prime}-f^{\prime c} \tilde{m}_{R R}^{2} \sigma_{\mu} \bar{f}^{\prime c}\right)\right]
$$

These four fermion terms can alternatively be extracted from the general formula $\Delta K=$ $\frac{\Lambda^{2}}{16 \pi^{2}} \log \operatorname{det} K_{\bar{m} n}$, which summarizes the quadratically divergent contribution of chiral supermultiplets to the Kähler potential [22].

We will use the effective lagrangian (23) to estimate the effect of flavour changing goldstino (or sgoldstino) interactions on $\Delta F=1$ processes such as $\mu \rightarrow e e e, K^{0} \rightarrow \ell \bar{\ell}$, $K \rightarrow \pi \bar{\ell}, \ldots$, or $\Delta F=2$ processes such as $K-\bar{K}$ transitions. As far as sfermion mass matrices are concerned, we will assume for simplicity that, in the fermion mass basis, the flavour diagonal entries in the $L L$ and $R R$ blocks have a common value $\tilde{m}^{2}$. We stress again that our results below should be regarded as indicative, not only because we are focusing on a specific class of contributions, but also because the quadratic sensitivity on the cutoff scale $\Lambda$ introduces a further uncertainty. Indeed, in the absence of information on the underlying theory, $\Lambda$ could either be a scale just above $\tilde{m}$ or take larger values, up to the scale where unitarity breaks down. Hence $\Lambda$ is expected to lie somewhere in the range $\left[\Lambda_{\min }, \Lambda_{\max }\right]$, where $\Lambda_{\min }^{2} \sim \tilde{m}^{2}$ and $\Lambda_{\max }^{2} \sim 16 \pi F^{2} / \tilde{m}^{2} \sim 16 \pi \tilde{\Lambda}^{2}$ [9, 12]. In the examples below, we will specialize our formulae to the two extreme values of $\Lambda$. We note that, for the conservative choice $\Lambda \sim \tilde{m}$, quadratically divergent contributions have similar parameter dependence and size as the other contributions (i.e. logarithmically divergent and finite ones), so in this case the former ones can also be interpreted as 'representatives' of the latter ones.

Consider for instance the terms in (23) that contribute to the lepton flavour violating process $\mu^{-} \rightarrow e^{-} e^{+} e^{-}$:

$$
\mathcal{L}_{e f f}=-\frac{1}{32 \pi^{2}} \frac{\tilde{m}^{2} \Lambda^{2}}{F^{4}}\left(\bar{e} \bar{\sigma}^{\mu} e-e^{c} \sigma^{\mu} \bar{e}^{c}\right)\left[\left(\tilde{m}_{L L}^{2}\right)_{e \mu} \bar{e} \bar{\sigma}_{\mu} \mu-\left(\tilde{m}_{R R}^{2}\right)_{\mu e} e^{c} \sigma_{\mu} \bar{\mu}^{c}\right]
$$

\footnotetext{
${ }^{13}$ The situation was slightly different in the computation of $\mu \rightarrow e \gamma$ presented in the previous section. In that case the operator was different, and our choice to only focus on couplings related to the spectrum led us to obtain a finite result. If we had included other terms in the Kähler potential or in the gauge kinetic function, however, we would also have obtained logarithmically divergent contributions, associated to a (supersymmetric) higher derivative term. For a more detailed discussion in the context of the flavour conserving magnetic moments, see [14].

${ }^{14}$ For a similar discussion about this and the previous points in the context of flavour conserving interactions, see 12 .
} 
If we focus on the part proportional to $\left(\tilde{m}_{L L}^{2}\right)_{e \mu}$ and neglect any other contribution to this process, we obtain:

$$
B R\left(\mu^{-} \rightarrow e^{-} e^{+} e^{-}\right) \simeq\left[\frac{\sqrt{6}}{128 \pi^{2}} \frac{\tilde{m}^{4} \Lambda^{2}}{G_{F} F^{4}}\left|\left(\delta_{L L}\right)_{e \mu}\right|\right]^{2}
$$

where $\left(\delta_{L L}\right)_{e \mu}=\left(\tilde{m}_{L L}^{2}\right)_{e \mu} / \tilde{m}^{2}$. Comparing the above expression with the experimental upper bound $10^{-12}$ [19] gives constraints on the parameters $\left(\delta_{L L}\right)_{e \mu}, \tilde{m}$ and $\sqrt{F}$, for a given $\Lambda$. For the two extreme choices of $\Lambda$, we obtain

$$
\begin{array}{ll}
\left|\left(\delta_{L L}\right)_{e \mu}\right| \lesssim 5 \times 10^{-4}\left(\frac{\sqrt{F}}{\tilde{m}}\right)^{8}\left(\frac{\tilde{m}}{300 \mathrm{GeV}}\right)^{2}, & \left(\Lambda=\Lambda_{\min }\right) \\
\left|\left(\delta_{L L}\right)_{e \mu}\right| \lesssim 10^{-5}\left(\frac{\sqrt{F}}{\tilde{m}}\right)^{4}\left(\frac{\tilde{m}}{300 \mathrm{GeV}}\right)^{2}, & \left(\Lambda=\Lambda_{\max }\right)
\end{array}
$$

If we take the smallest value of $\Lambda$, the constraints on $\left(\delta_{L L}\right)_{e \mu}$ are comparable or stronger than those obtained in the previous section from the analysis of goldstino contributions to $\mu \rightarrow e \gamma$. This can be seen, for instance, by comparing eq. (26) with eq. (21) (also notice the different dependence on $\sqrt{F} / \tilde{m})$. If we take the largest possible value of $\Lambda$, the constraints on $\left(\delta_{L L}\right)_{e \mu}$ are even stronger, for fixed values of the other parameters. We recall that for large $\sqrt{F}$, i.e. when the non-goldstino contributions are the dominant ones, the process $\mu \rightarrow e \gamma$ is more sensitive to $\left(\delta_{L L}\right)_{e \mu}$ as compared to $\mu \rightarrow$ eee [17]. Here we have found that when $\sqrt{F}$ approaches $\tilde{m}$, i.e. when goldstino contributions become important, the situation may be reversed. Similar considerations apply to $\left(\delta_{R R}\right)_{e \mu}$, of course.

The lepton flavour violating decay $\pi^{0} \rightarrow \mu e$ is another process that is sensitive to $\left(\tilde{m}_{L L}^{2}\right)_{e \mu}$ and $\left(\tilde{m}_{R R}^{2}\right)_{e \mu}$. This decay receives contributions from terms in (23) which couple a muon-electron current to up or down quark currents. The latter currents couple to the pion provided the up and down squark masses are non-degenerate. However, owing to the rapidity of the dominant decay $\pi^{0} \rightarrow \gamma \gamma$, no significant constraints are obtained on $\left(\delta_{L L}\right)_{e \mu}$ and $\left(\delta_{R R}\right)_{e \mu}$. Another process potentially sensitive to the latter quantities is the $\mu \rightarrow e$ conversion on nuclei. The effective interactions in (23) also contribute to other lepton flavour violating processes, such as $\tau$ decays into either three charged leptons or a charged lepton and a $\pi^{0}$. These processes are sensitive to flavour changing slepton masses involving the third generation, but no strong constraints are obtained.

In the final part of this section, we consider some examples of flavour violation in the quark sector induced by the four-fermion terms in (23). In particular, we focus on two processes that are sensitive to the $s d$ entries of the squark mass matrix, i.e. the decay $K_{L} \rightarrow \mu^{+} \mu^{-}(\Delta S=1)$ and $K-\bar{K}$ transitions $(\Delta S=2)$. Since we are dealing with order-of-magnitude estimates, we neglect $Q C D$ corrections and use the vacuum insertion approximation. The terms in (23) that contribute to the decay $K_{L} \rightarrow \mu^{+} \mu^{-}$are

$$
\mathcal{L}_{e f f}=-\frac{1}{32 \pi^{2}} \frac{\tilde{m}^{2} \Lambda^{2}}{F^{4}}\left(\bar{\mu} \bar{\sigma}^{\mu} \mu-\mu^{c} \sigma^{\mu} \bar{\mu}^{c}\right)\left[\left(\tilde{m}_{L L}^{2}\right)_{d s} \bar{d} \bar{\sigma}_{\mu} s-\left(\tilde{m}_{R R}^{2}\right)_{d s} d^{c} \sigma_{\mu} \bar{s}^{c}+\text { h.c. }\right]
$$

\footnotetext{
${ }^{15}$ These terms or analogous ones (with the muon replaced by another lepton or a quark) also contribute to other $\Delta S=1$ decays, such as $K \rightarrow \pi \bar{\ell}$ or $K \rightarrow \pi \pi$ (hence $\epsilon^{\prime}$ ).
} 
If we only consider the part proportional to $\left(\tilde{m}_{L L}^{2}\right)_{d s}$, we obtain:

$$
B R\left(K_{L} \rightarrow \mu^{+} \mu^{-}\right) \simeq \xi \cdot\left[\frac{\sqrt{2}}{32 \pi^{2}} \frac{\tilde{m}^{4} \Lambda^{2}}{\sin \theta_{c} G_{F} F^{4}} \operatorname{Re}\left(\delta_{L L}\right)_{d s}\right]^{2}
$$

where $\left(\delta_{L L}\right)_{d s}=\left(\tilde{m}_{L L}^{2}\right)_{d s} / \tilde{m}^{2}, \xi \equiv B R\left(K^{+} \rightarrow \mu^{+} \nu_{\mu}\right) \tau\left(K_{L}\right) / \tau\left(K^{+}\right) \simeq 2.7$ and $\theta_{c}$ is the Cabibbo angle. By imposing that the value of the above expression does not exceed the observed value $\left.7 \times 10^{-9} \llbracket 19\right]$, we can obtain combined constraints on $\left|\operatorname{Re}\left(\delta_{L L}\right)_{d s}\right|, \tilde{m}$ and $\sqrt{F}$, for a given $\Lambda$. For the two extreme choices of $\Lambda$, we obtain

$$
\begin{array}{ll}
\left|\operatorname{Re}\left(\delta_{L L}\right)_{d s}\right| \lesssim 3 \times 10^{-3}\left(\frac{\sqrt{F}}{\tilde{m}}\right)^{8}\left(\frac{\tilde{m}}{300 \mathrm{GeV}}\right)^{2}, \quad\left(\Lambda=\Lambda_{\min }\right) \\
\left|\operatorname{Re}\left(\delta_{L L}\right)_{d s}\right| \lesssim 6 \times 10^{-5}\left(\frac{\sqrt{F}}{\tilde{m}}\right)^{4}\left(\frac{\tilde{m}}{300 \mathrm{GeV}}\right)^{2}, \quad\left(\Lambda=\Lambda_{\max }\right)
\end{array}
$$

We now consider the terms in (23) that contribute to $K-\bar{K}$ transitions:

$$
\mathcal{L}_{e f f}=-\frac{1}{64 \pi^{2}} \frac{\Lambda^{2}}{F^{4}}\left[\left(\tilde{m}_{L L}^{2}\right)_{d s} \bar{d} \bar{\sigma}^{\mu} s-\left(\tilde{m}_{R R}^{2}\right)_{d s} d^{c} \sigma^{\mu} \bar{s}^{c}\right]\left[\left(\tilde{m}_{L L}^{2}\right)_{d s} \bar{d} \bar{\sigma}_{\mu} s-\left(\tilde{m}_{R R}^{2}\right)_{d s} d^{c} \sigma_{\mu} \bar{s}^{c}\right]+\text { h.c. }
$$

Retaining again only the $L L$ contributions, we obtain:

$$
\left|\frac{\Delta m_{K}}{m_{K}}\right| \simeq \frac{1}{96 \pi^{2}} \frac{f_{K}^{2} \tilde{m}^{4} \Lambda^{2}}{F^{4}}\left|\operatorname{Re}\left(\delta_{L L}\right)_{d s}^{2}\right|
$$

where $f_{K} \simeq 160 \mathrm{MeV}$ is the kaon decay constant. We can again find constraints on the parameters by imposing that the value of the above expression does not exceed the experimental value $7 \times 10^{-15}$. For the two extreme choices of $\Lambda$, we obtain

$$
\begin{array}{ll}
\sqrt{\left|\operatorname{Re}\left(\delta_{L L}\right)_{d s}^{2}\right|} \lesssim 5 \times 10^{-3}\left(\frac{\sqrt{F}}{\tilde{m}}\right)^{4}\left(\frac{\tilde{m}}{300 \mathrm{GeV}}\right), \quad\left(\Lambda=\Lambda_{\min }\right) \\
\sqrt{\left|\operatorname{Re}\left(\delta_{L L}\right)_{d s}^{2}\right|} \lesssim 7 \times 10^{-4}\left(\frac{\sqrt{F}}{\tilde{m}}\right)^{2}\left(\frac{\tilde{m}}{300 \mathrm{GeV}}\right), \quad\left(\Lambda=\Lambda_{\max }\right)
\end{array}
$$

When $\sqrt{F} \gg \tilde{m}$, the above eqs. (30-31) and (34 35) do not give significant bounds on $\left(\delta_{L L}\right)_{d s}$, which is instead constrained by the non-goldstino contributions. We recall that, in this case, the strongest bound comes from $\Delta m_{K}$ rather than $K_{L} \rightarrow \mu^{+} \mu^{-}$, since only the former quantity receives significant contributions from diagrams with gluino exchange. When $\sqrt{F}$ approaches $\tilde{m}$, on the other hand, the goldstino contributions become more and more relevant and the limit on $\left(\delta_{L L}\right)_{d s}$ obtained from $K_{L} \rightarrow \mu^{+} \mu^{-}$can be comparable or even more stringent than that from $\Delta m_{K}$.

The quantity $\left(\delta_{R R}\right)_{d s}$ is constrained in the same way as $\left(\delta_{L L}\right)_{d s}$. The bounds from $\Delta m_{K}$ on the combination $\sqrt{\left|\operatorname{Re}\left[\left(\delta_{L L}\right)_{d s}\left(\delta_{R R}\right)_{d s}\right]\right|}$ are slightly more stringent than those in eqs. (34. 35). The $\Delta S=2$ lagrangian in eq. (32) also contributes to the $\mathrm{CP}$ violating parameter $\epsilon_{K}$. The resulting bounds on $\sqrt{\left|\operatorname{Im}\left(\delta_{L L}\right)_{d s}^{2}\right|}, \sqrt{\left|\operatorname{Im}\left(\delta_{R R}\right)_{d s}^{2}\right|}$ and $\sqrt{\mid \operatorname{Im}\left[\left(\delta_{L L}\right)_{d s}\left(\delta_{R R}\right)_{d s}||\right.}$ are about an order of magnitude smaller than those of the corresponding real parts. 
Flavour violating processes involving $B(D)$ mesons can be discussed along similar lines. The bounds on $\left(\delta_{L L}\right)_{d b}\left(\left(\delta_{L L}\right)_{u c}\right)$ from $B_{d^{-}} \bar{B}_{d}(D-\bar{D})$ mixing, for instance, are slightly weaker than the corresponding ones in the $K-\bar{K}$ system. The effective lagrangian (23) also contributes to flavour changing processes involving external top quarks, if the appropriate entries in $\tilde{m}_{L L}^{2}$ or $\tilde{m}_{R R}^{2}$ are non-vanishing. Moreover, the latter processes can also be sensitive to the off-diagonal entries of $\tilde{m}_{R L}^{2}$ related to the top, since the chiral suppression is less effective. In this respect, even the effective interactions due to tree-level sgoldstino exchange (see eq. (22) and related paragraph) can play a role in decays such as $t \rightarrow c f \bar{f}$ or $t \rightarrow c \gamma \gamma$.

\section{Flavour changing processes with external goldstinos}

Up to now we have discussed flavour changing processes that have photons and ordinary fermions (leptons and quarks) as external states, with goldstinos, sgoldstinos, sleptons, squarks and photinos present in internal lines only. Now we will consider the possibility that the external states also include goldstinos, but (again) not the other superpartners, which we integrate out. In particular, we would like to discuss whether flavour violations can occur in low-energy processes involving two ordinary fermions and two goldstinos, i.e. whether transitions such as $\mu \rightarrow e \tilde{G} \tilde{G}$ or $s \rightarrow d \tilde{G} \tilde{G}$ can take place.

We first study what happens when sfermions and sgoldstinos are integrated out at tree-level. Using the masses and couplings described in Section 2, we find that three types of diagrams contribute (see Fig. 5). We recall that vertices and sfermion propagators have

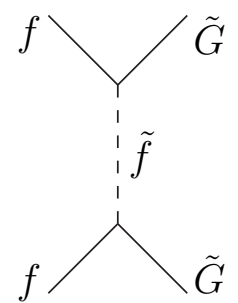

(a)

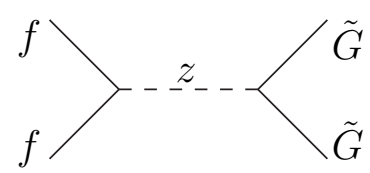

(b)

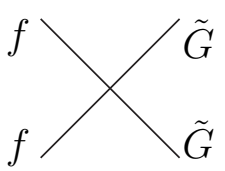

(c)

Figure 5: Tree-level diagrams contributing to effective interactions between two goldstinos and two matter fermions. The symbols $f$ and $\tilde{f}$ generically denote matter fermions and sfermions.

a non-trivial flavour structure. However, such structures combine in a characteristic way if we expand the scalar propagators around the heavy (supersymmetry breaking) scalar masses and treat momenta and fermion masses (i.e. $\square$ and $\mathrm{mm}^{\dagger}$ terms) as perturbations. Indeed, we obtain ${ }^{16}$ :

$$
(a) \Longrightarrow \frac{1}{F^{2}}\left(\begin{array}{cc}
\overline{\tilde{G}} \bar{f} & \tilde{G} f^{c}
\end{array}\right)\left(\begin{array}{cc}
\tilde{m}_{L L}^{2} & \tilde{m}_{L R}^{2} \\
\tilde{m}_{R L}^{2} & \tilde{m}_{R R}^{2}
\end{array}\right)\left(\begin{array}{c}
f \tilde{G} \\
\bar{f} c \overline{\tilde{G}}
\end{array}\right)+\ldots
$$

\footnotetext{
${ }^{16}$ This holds for each fermion species $f$, of course: we omit the sum $\sum_{f}$ for brevity. Notice that generation (i.e. flavour) indices are still understood.
} 


$$
\begin{aligned}
& (b) \Longrightarrow-\frac{1}{F^{2}}\left(\begin{array}{cc}
\overline{\tilde{G}} \bar{f} & \tilde{G} f^{c}
\end{array}\right)\left(\begin{array}{cc}
0 & \tilde{m}_{L R}^{2} \\
\tilde{m}_{R L}^{2} & 0
\end{array}\right)\left(\begin{array}{c}
f \tilde{G} \\
\bar{f}^{c} \tilde{G}
\end{array}\right)+\ldots \\
& (c) \Longrightarrow-\frac{1}{F^{2}}\left(\begin{array}{cc}
\overline{\tilde{G}} \bar{f} & \tilde{G} f^{c}
\end{array}\right)\left(\begin{array}{cc}
\tilde{m}_{L L}^{2} & 0 \\
0 & \tilde{m}_{R R}^{2}
\end{array}\right)\left(\begin{array}{c}
f \tilde{G} \\
\bar{f}^{c} \tilde{G}
\end{array}\right)
\end{aligned}
$$

where the dots denote terms suppressed by powers of momenta or fermion masses. We immediately see that, once we sum the leading terms from sfermion (a) and sgoldstino (b) exchange with the contact term (c), a complete cancellation takes place, as it should. The first nonvanishing contributions arise at the next order in the expansion, and are quadratic in momenta or fermion masses. In particular, from sfermion diagrams we obtain

$$
\begin{aligned}
(a) \Longrightarrow & -\frac{1}{F^{2}}\left[\overline{\tilde{G}} \bar{f}\left(\square+m^{\dagger} m\right) f \tilde{G}+\tilde{G} f^{c}\left(\square+m m^{\dagger}\right) \bar{f}^{c} \tilde{G}\right] \\
& =-\frac{2}{F^{2}}(\overline{\tilde{G}} \bar{f})\left(\partial^{\mu} f \partial_{\mu} \tilde{G}\right)+\left(f \rightarrow f^{c}\right)
\end{aligned}
$$

where we have used the equations of motion to write the second expression. Contributions quadratic in momenta also come from sgoldstino diagrams (b). However, these operators also contain factors like $\tilde{m}_{R L}^{2} / m_{S}^{2}$ or $\tilde{m}_{R L}^{2} / m_{P}^{2}$, so they can be considered of higher order, under the assumption that $\tilde{m}_{R L}^{2}$ is chirally suppressedШ (i.e. linear in fermion masses). So, according to this procedure, the leading non-vanishing interaction between on-shell goldstinos and matter fermions is the dimension 8 operator in eq. (39). Notice that this operator is manifestly flavour universal and does not depend on the superpartner spectrum. In particular, no trace remains of the flavour structure of sfermion mass matrices.

This result generalizes that obtained in the one-flavour case by a similar procedure [9, 10. On the other hand, the effective low-energy interactions between goldstinos and matter fermions can also be obtained by other methods, e.g. by direct non-linear realizations of supersymmetry [23], which do not require the explicit introduction of superpartners. In such frameworks, a more general result can be obtained. In the one-flavour case, for instance, it was recently shown [10, 11] that the on-shell interactions between two goldstinos and two $f$-type fermions are described by two independent operators:

$$
\mathcal{L}_{n l}=-\frac{1}{F^{2}}\left[\left(\overline{\tilde{G}} \bar{\sigma}^{\mu} \partial_{\nu} \tilde{G}\right)\left(\bar{f} \bar{\sigma}^{\nu} \partial_{\mu} f\right)-\left(\bar{f} \bar{\sigma}^{\mu} \partial_{\nu} \tilde{G}\right) C^{(f)}\left(\partial_{\mu} \overline{\tilde{G}} \bar{\sigma}^{\nu} f\right)\right]
$$

where $C^{(f)}$ is an arbitrary dimensionless coefficient 18 . This result can be easily generalized to the multi-flavour case: we only need to reinterpret $f$ as a collection of fermions $f_{i}$ and the coefficient $C^{(f)}$ as a matrix in flavour space, $C^{(f)}=C_{i j}^{(f)}$. The operators for the fermions $f^{c}$ are analogous, with a matrix $C^{\left(f^{c}\right)}$. We recall that the first operator in (40) corresponds to the standard coupling of goldstinos to the energy-momentum tensor [23]. Notice that it is flavour universal, but differs from the operator in eq. (39). Flavour violations can only come from the second operator in (40), if the matrix $C^{(f)}$ is not diagonal in the fermion mass basis. Before discussing this possibility, it is convenient to write eq. (40) in two

\footnotetext{
${ }^{17}$ We recall that such a suppression could be rather mild if the top quark is involved. However, here (as before) we are mainly interested in low-energy processes, where only light fermions are involved.

18 The normalization we have chosen is such that $C^{(f)}=-\frac{1}{4} \alpha 10=\frac{1}{2} C_{f f}[1]$.
} 
additional equivalent forms. Using Fierz rearrangements and the goldstino equations of motion, we obtain:

$$
\begin{aligned}
\mathcal{L}_{n l} & =-\frac{2}{F^{2}}\left[(\overline{\tilde{G}} \bar{f})\left(\partial^{\mu} f \partial_{\mu} \tilde{G}\right)+\left(\partial^{\mu} \overline{\tilde{G}} \bar{f}\right)\left(1-C^{(f)}\right)\left(f \partial_{\mu} \tilde{G}\right)\right] \\
& =-\frac{2}{F^{2}}\left[(\overline{\tilde{G}} \bar{f})\left(\partial^{\mu} f \partial_{\mu} \tilde{G}\right)+\frac{1}{4}\left(\bar{f}\left(1-C^{(f)}\right) \bar{\sigma}^{\mu} f\right) \square\left(\overline{\tilde{G}} \bar{\sigma}_{\mu} \tilde{G}\right)\right]
\end{aligned}
$$

where 11 is the unity matrix in flavour space. In particular, by comparing eq. (41) with the interaction found by integrating out superpartners, eq. (39), we can see that the latter corresponds to the special case $C^{(f)}=11$. In the one-flavour case, an analogous result was obtained in [10] by explicit computation and comparison of scattering amplitudes.

We have seen that the non-linear formulation of spontaneously broken supersymmetry allows for a generic, flavour non-universal matrix $C^{(f)}$. On the other hand, integrating out superpartners from an effective theory with linearly realized supersymmetry has led us to find a specific, universal $C^{(f)}$. Therefore we can wonder whether a non-universal $C^{(f)}$ could emerge also in the effective linear approach, by generalizing the decoupling procedure and/or the theory itself. We will now mention a few such possibilities.

(i) One possibility could be to keep using the structure described in Section 2, and then perform the decoupling of superpartners at one-loop level, rather than at tree-level. Since the full computation is quite involved, we could first focus on the quadratically divergent contributions only. If we do this, however, we find that the final result still has the form (39), which corresponds to a universal $C^{(f)}$. This follows from the fact that the inclusion of such corrections amounts to use a corrected Kähler potential. Thus, once the theory is expressed in terms of one-loop corrected fields, masses and couplings, the decoupling procedure for the interactions under study is formally similar to the tree-level one. However, this argument does not necessarily hold for logarithmically divergent and finite corrections, where the flavour structure of sfermion mass matrices might survive and lead to non-universal contributions to $C^{(f)}$. Notice that the superspace interpretation of those corrections corresponds to both Kähler and non-Kähler (i.e. higher derivative) terms. These considerations also suggest a different (alternative) approach, in which computations are done at the tree level, by starting however from an effective lagrangian which already contains higher derivative terms, besides the Kählerian ones.

(ii) Another possibility could be to relax the assumption of pure F-type supersymmetry breaking used so far. We can consider a more general situation, with larger field content and gauge structure, such that non-vanishing auxiliary component vevs appear in both chiral and vector supermultiplets (mixed $F$ - $D$ breaking). In this case, the goldstino is a linear combination of the fermions in those multiplets, and the sgoldstino sector includes the bosonic components of such multiplets, i.e. both spin-0 fields (like $z$ above) and spin-1 fields (see e.g. [24, 25]). Sfermion masses receive both $F$-type and $D$-type supersymmetry breaking contributions. Once all this is taken into account, one can again integrate out sfermions and sgoldstinos at tree level and find the effective interactions between two matter fermions and two goldstinos. We have checked that the leading terms again cancel, as they should 
(see also 25]). At first non-vanishing order we find dimension 8 operators as in eqs. (41-42). Diagrams with sfermion exchange again give a universal contribution to $C^{(f)}$ (the same as before). Diagrams with spin-1 sgoldstino exchange give an additional model dependent contribution to $C^{(f)}$. The latter contribution depends e.g. on the coupling (charges) of matter fermions with spin-1 sgoldstinos. If these charges are neither universal nor aligned with fermion masses, flavour changing effects can arise. In this case, however, one should also keep under control the contributions of spin-1 sgoldstino exchange to (dimension 6) flavour changing operators involving four matter fermions.

Exploring in more detail the possibilities mentioned above, or other ones, lies beyond the scope of the present paper. For the rest of our discussion, we will rely on the fact that supersymmetry in principle allows for the existence of effective (two goldstino)-(two matter fermion) operators with non-diagonal matrices $C^{(f)}$, and ask what this could imply for phenomenology. We will see that the high dimensionality of the effective operators implies by itself a strong suppression, so that even low values of $\sqrt{F}$ and large flavour violating entries in $C^{(f)}$ are allowed. Consider for instance the charged lepton sector, and assume that $C_{e \mu}$ and/or $C_{e^{c} \mu^{c}}$ are non-vanishing, so that the flavour changing decay $\mu^{-} \rightarrow e^{-} \tilde{G} \tilde{G}$ can take place. Although this decay is flavour changing, the final state is very similar to that of the flavour conserving decay $\mu^{-} \rightarrow e^{-} \nu_{\mu} \bar{\nu}_{e}$, which proceeds at leading order through the standard Fermi interaction. The corresponding operators can be cast into similar forms (see eq. (42)):

$$
\begin{gathered}
\frac{1}{2 F^{2}}\left[C_{e \mu}\left(\bar{e} \bar{\sigma}^{\mu} \mu\right)-C_{\mu^{c} e^{c}}\left(e^{c} \sigma^{\mu} \bar{\mu}^{c}\right)\right] \square\left(\tilde{\tilde{G}} \bar{\sigma}_{\mu} \tilde{G}\right) \\
2 \sqrt{2} G_{F}\left(\bar{e} \bar{\sigma}^{\mu} \mu\right)\left(\bar{\nu}_{\mu} \bar{\sigma}_{\mu} \nu_{e}\right)
\end{gathered}
$$

The presence of two derivatives in the first operator, however, gives a strong suppression. Indeed, the ratio of the two decay rates scales as:

$$
\frac{\Gamma\left(\mu^{-} \rightarrow e^{-} \tilde{G} \tilde{G}\right)}{\Gamma\left(\mu^{-} \rightarrow e^{-} \nu_{\mu} \bar{\nu}_{e}\right)} \sim\left(\left|C_{e \mu}\right|^{2}+\left|C_{\mu^{c} e^{c}}\right|^{2}\right) \frac{m_{\mu}^{4}}{G_{F}^{2} F^{4}}
$$

Even if we take $F \simeq G_{F}^{-1}$ and $\left|C_{e \mu}\right|^{2}+\left|C_{e^{c} \mu^{c}}\right|^{2}=\mathcal{O}(1)$, the branching ratio is tiny, $\mathcal{O}\left(10^{-13}\right)$. Although the features of the electron emitted with the goldstino pair (polarization, energy and angular distributions) differ from those of the standard channel, the numerical suppression is so strong that detection seems impossible. Similar considerations apply if we compare the decays $\tau \rightarrow e \tilde{G} \tilde{G}$ and $\tau \rightarrow \mu \tilde{G} \tilde{G}$ to the corresponding standard ones. Even though $m_{\mu}^{4}$ above is replaced by $m_{\tau}^{4}$, the ratios analogous to (45) are still tiny, e.g. $\mathcal{O}\left(10^{-9}\right)$ if $F \simeq G_{F}^{-1}$ and the $C_{i j}$ are $\mathcal{O}(1)$.

We finally consider flavour changing transitions with goldstino pair emission in the quark sector. Consider for instance the operator

$$
\frac{1}{2 F^{2}}\left[C_{s d}\left(\bar{s} \bar{\sigma}^{\mu} d\right)-C_{d^{c} s^{c}}\left(s^{c} \sigma^{\mu} \bar{d}^{c}\right)\right] \square\left(\tilde{\tilde{G}} \bar{\sigma}_{\mu} \tilde{G}\right)
$$


This operator does not contribute to $K^{0} \rightarrow \tilde{G} \tilde{G}$, but does contribute e.g. to $K^{+} \rightarrow \pi^{+} \tilde{G} \tilde{G}$. This is similar to the decay $K^{+} \rightarrow \pi^{+} \nu \bar{\nu}$, which is itself very suppressed when compared to its charged counterpart. For instance, in the SM one expects (see e.g. 26])

$$
\frac{\Gamma\left(K^{+} \rightarrow \pi^{+} \nu \bar{\nu}\right)}{\Gamma\left(K^{+} \rightarrow \pi^{0} e^{+} \nu_{e}\right)} \sim 10^{-9}
$$

For $K^{+} \rightarrow \pi^{+} \tilde{G} \tilde{G}$, the corresponding ratio scales as

$$
\frac{\Gamma\left(K^{+} \rightarrow \pi^{+} \tilde{G} \tilde{G}\right)}{\Gamma\left(K^{+} \rightarrow \pi^{0} e^{+} \nu_{e}\right)} \sim\left(\left|C_{s d}\right|^{2}+\left|C_{d^{c} s^{c}}\right|^{2}\right) \frac{m_{K}^{4}}{G_{F}^{2} F^{4}}
$$

Even in the extreme case in which $F \simeq G_{F}^{-1}$ and $\left|C_{d s}\right|$ (or $\left|C_{s^{c} d^{c}}\right|$ ) is $\mathcal{O}(1)$, the latter ratio is $\mathcal{O}\left(10^{-11}\right)$, which is smaller than the ratio in (47). The rates for the analogous $B$ decays $(B \rightarrow \pi \tilde{G} \tilde{G}, B \rightarrow K \tilde{G} \tilde{G})$ are also smaller than the corresponding ones with neutrino pair emission. To weaken the effect of the low-energy suppression, we could move to higher energies and consider the top quark. Operators analogous to those discussed above could induce, for example, the flavour changing decay $t \rightarrow c \tilde{G} \tilde{G}$. The corresponding rate would be strongly enhanced by the presence of $m_{t}$ : if $F \simeq G_{F}^{-1}$ and $\left|C_{t c}\right|$ (or $\left|C_{t^{c} c^{c}}\right|$ ) is $\mathcal{O}(1)$, $B R(t \rightarrow c \tilde{G} \tilde{G})$ could reach values as large as $\mathcal{O}\left(10^{-2}\right)$.

\section{Summary}

In this paper we have pointed out and discussed a new source of flavour violation in supersymmetric models, namely the couplings of goldstinos with matter. Since those couplings are strictly related to the mass spectrum and are suppressed by the supersymmetry breaking scale $\sqrt{F}$, significant effects on FCNC processes can be obtained when the following two ingredients are present: (i) the sfermion mass matrices have a non-trivial flavour structure in the fermion mass basis; (ii) the supersymmetry breaking scale $\sqrt{F}$ is not much larger than the electroweak scale. Notice that condition (i) is the same feature that is responsible for the well-known flavour changing effects induced by gaugino-matter couplings. In the latter case, the effects are enhanced when the supersymmetry breaking masses $\tilde{m}$ are close to the electroweak scale: point (ii) expresses the analogous property for goldstino contributions. In other words, for given flavour violating sfermion mass matrices, goldstino and non-goldstino contributions to FCNC become comparable when $\sqrt{F}$ and $\tilde{m}$ have a similar size.

These considerations especially apply to the usual class of low-energy FCNC processes, which involve photons, leptons and quarks in the external states. In this case, goldstinos (or sgoldstinos) can contribute as virtual particles, as well as sleptons, squarks and gauginos. In Section 3 and Section 4 we have examined the sensitivity of several such processes to the value of $\sqrt{F}$ and to the amount of flavour violation in the sfermion masses (parametrized by the popular quantities $\delta_{i j}$ ), also making comparisons with the conventional non-goldstino contributions. In Section 3 we have discussed the decay $\mu \rightarrow e \gamma$ as a prototype of flavour changing radiative decays. The analysis confirms that, when $\sqrt{F}$ and $\tilde{m}$ (or $M$ ) have a similar size, the contributions from goldstino-slepton-photino 
exchange become comparable to those from slepton-photino exchange and can interfere either constructively or destructively (see Fig. 2). When goldstino contributions dominate, the bounds on $\delta_{e \mu}$ become stronger than the conventional ones, for given $(\tilde{m}, M)$ (see Fig. 3). A similar picture has emerged from the analysis of flavour violating processes with four external matter fermions, discussed in Section 4. In this case we have focused on a representative class of goldstino (sgoldstino) contributions, which we have used to obtain order-of-magnitude estimates, taking also into account the uncertainty due to the cutoff scale $\Lambda$. In this context we have first considered the decay $\mu \rightarrow e e e$. Again, when $\sqrt{F} \sim \tilde{m}$ the contributions due to the goldstino multiplet become comparable or even more important than those from photino-slepton exchange. In this situation, moreover, the decay $\mu \rightarrow$ eee seems to have similar or even stronger sensitivity to $\delta_{e \mu}$ as compared to $\mu \rightarrow e \gamma$, contrary to what happens in the conventional scenario (which corresponds to $\sqrt{F} \gg \tilde{m}$ ). In the quark sector, we have discussed processes such as $K_{L} \rightarrow \mu^{+} \mu^{-}$ and $K-\bar{K}$ transitions. Again, the goldstino contributions can become dominant for low values of $\sqrt{F}$. In this limit, the bounds on the parameters $\delta_{d s}$ from $K_{L} \rightarrow \mu^{+} \mu^{-}$can be comparable or even more stringent than those from $\Delta m_{K}$.

Finally, in Section 5, we have considered processes with two matter fermions and two goldstinos as external states. The corresponding four-fermion operators have effective dimension 8 rather than 6 , due to the special low-energy properties of goldstinos. When we have obtained such operators by integrating out heavy superpartners at tree-level, that feature has emerged because of mutual cancellations among the (otherwise leading) dimension 6 terms. By using this procedure, however, we have found that the resulting operators do not exhibit any flavour structure. The latter result is by itself quite remarkable. On the other hand, it cannot be regarded as completely general. Indeed, we have noted that the more general context of non-linearly realized supersymmetry in principle allows for operators with a non trivial flavour dependence. We have discussed how this result might be recovered in the linear approach, e.g. by considering the case of mixed $F-D$ supersymmetry breaking rather than pure $F$-breaking. As regards the phenomenological implications, we have seen that, even in the presence of flavour violating couplings, the high dimensionality of the effective operators automatically suppresses potentially interesting transitions with goldstino pair-emission. For instance, even in the case of maximal flavour violation and $\sqrt{F} \sim\left(G_{F}\right)^{-1 / 2}$, meson decays such as $K \rightarrow \pi \tilde{G} \tilde{G}$ have smaller rates as compared to $K \rightarrow \pi \nu \bar{\nu}$.

In conclusion, our general analysis shows that low-energy FCNC processes are sensitive probes of supersymmetric scenarios that involve both a low supersymmetry breaking scale and some amount of flavour violation in the sfermion sector. It would be very interesting to see how both features could emerge in concrete models at a more fundamental level.

Acknowledgements. We thank F. Feruglio, A. Masiero and F. Zwirner for discussions. 


\section{References}

[1] S. L. Glashow, J. Iliopoulos and L. Maiani, Phys. Rev. D2 (1970) 1285;

M. K. Gaillard and B. W. Lee, Phys. Rev. D10 (1974) 897.

[2] S. Dimopoulos and H. Georgi, Nucl. Phys. B193 (1981) 150;

J. Ellis and D. V. Nanopoulos, Phys. Lett. B110 (1982) 44;

R. Barbieri and R. Gatto, Phys. Lett. B110 (1982) 211;

T. Inami and C. S. Lim, Nucl. Phys. B207 (1982) 533;

M. J. Duncan, Nucl. Phys. B221 (1983) 285;

J. F. Donoghue, H. P. Nilles and D. Wyler, Phys. Lett. B128 (1982) 55.

[3] F. Gabbiani and A. Masiero, Nucl. Phys. B322 (1989) 235.

[4] J. S. Hagelin, S. Kelley and T. Tanaka, Nucl. Phys. B415 (1994) 293;

F. Gabbiani, E. Gabrielli, A. Masiero and L. Silvestrini, Nucl. Phys. B477 (1996) 321.

[5] P. Fayet, Phys. Lett. B70 (1977) 461.

[6] R. Casalbuoni, S. De Curtis, D. Dominici, F. Feruglio and R. Gatto, Phys. Lett. B215 (1988) 313 and Phys. Rev. D39 (1989) 2281.

[7] A. Brignole, F. Feruglio and F. Zwirner, Nucl. Phys. B516 (1998) $13+$ (E) B555 (1999) 653;

A. Brignole, F. Feruglio, M. L. Mangano and F. Zwirner, Nucl. Phys. B526 (1998) 136 and erratum hep-ph/9801329].

[8] G. F. Giudice and R. Rattazzi, Phys. Rept. 322 (1999) 419.

[9] A. Brignole, F. Feruglio and F. Zwirner, Nucl. Phys. B501 (1997) 332.

[10] A. Brignole, F. Feruglio and F. Zwirner, JHEP 9711 (1997) 001.

[11] T. E. Clark, T. Lee, S. T. Love and G. Wu, Phys. Rev. D57 (1998) 5912.

[12] A. Brignole, F. Feruglio and F. Zwirner, Phys. Lett. B438 (1998) 89.

[13] D. A. Dicus and S. Nandi, Phys. Rev. D56 (1997) 4166;

J. A. Grifols, R. N. Mohapatra and A. Riotto, Phys. Lett. B400 (1997) 124;

T. Gherghetta, Phys. Lett. B423 (1998) 311.

[14] A. Brignole, E. Perazzi and F. Zwirner, JHEP 9909 (1999) 002.

[15] E. Perazzi, G. Ridolfi and F. Zwirner, Nucl. Phys. B574 (2000) 3 and hep-ph/0005076.

[16] R. Barbieri and L. Maiani, Phys. Lett. B117 (1982) 203;

F. del Aguila, Phys. Lett. B160 (1985) 87.

[17] F. Borzumati and A. Masiero, Phys. Rev. Lett. 57 (1986) 961. 
[18] M. L. Brooks et al. [MEGA Collaboration], Phys. Rev. Lett. 83 (1999) 1521.

[19] C. Caso et al., Eur. Phys. J. C3 (1998) 1.

[20] S. Ahmed et al. [CLEO Collaboration], Phys. Rev. D61 (2000) 071101.

[21] S. Bertolini, F. Borzumati and A. Masiero, Phys. Lett. B192 (1987) 437.

[22] M. T. Grisaru, M. Rocek and A. Karlhede, Phys. Lett. B120 (1983) 110.

[23] D. V. Volkov and V. P. Akulov, Phys. Lett. B46 (1973) 109;

S. Samuel and J. Wess, Nucl. Phys. B221 (1983) 153.

[24] P. Fayet, Phys. Lett. B69 (1977) 489 and B84 (1979) 416.

[25] P. Fayet, Phys. Lett. B86 (1979) 272, B117 (1982) 460 and B175 (1986) 471.

[26] G. Buchalla, A. J. Buras and M. E. Lautenbacher, Rev. Mod. Phys. 68 (1996) 1125. 